\title{
External Iliac Artery Injury and Thrombosis during Laparoscopic Gynecologic Surgery
}

\author{
Masako Ishikawa, ${ }^{1}$ Kentaro Nakayama ${ }^{D},{ }^{1}$ Sultana Razia, ${ }^{1}$ Hitomi Yamashita, ${ }^{1}$ \\ Tomoka Ishibashi, ${ }^{1}$ Seiya Sato, ${ }^{1}$ Hiroki Sasamori, ${ }^{1}$ Kiyoka Sawada, ${ }^{1}$ Sonomi Kurose, ${ }^{1}$ \\ Noriyoshi Ishikawa, ${ }^{2}$ Shoichi Suehiro, ${ }^{3}$ and Satoru Kyo ${ }^{1}$ \\ ${ }^{1}$ Department of Obstetrics and Gynecology, Faculty of Medicine, Shimane University, 6938501 Izumo, Japan \\ ${ }^{2}$ Department of Organ Pathology, Faculty of Medicine, Shimane University, 6938501 Izumo, Japan \\ ${ }^{3}$ Division of Cardiovascular and Thoracic Surgery, Department of Surgery, Faculty of Medicine, Shimane University, \\ 6938501 Izumo, Japan
}

Correspondence should be addressed to Kentaro Nakayama; kn88@med.shimane-u.ac.jp

Received 8 April 2020; Revised 15 October 2020; Accepted 26 October 2020; Published 11 November 2020

Academic Editor: Cem Ficicioglu

Copyright (c) 2020 Masako Ishikawa et al. This is an open access article distributed under the Creative Commons Attribution License, which permits unrestricted use, distribution, and reproduction in any medium, provided the original work is properly cited.

\begin{abstract}
Although vascular injury during laparoscopic surgery is rare, it is occasionally reported. Previously, several physicians have reported cases of compartment syndrome resulting from a thrombus during gynecologic surgery. However, few reports describe thrombosis occurring in artery vessels as a result of applying angiopressure. Herein, we report the case of a 53-year-old woman with endometrioid carcinoma and a vascular injury that resulted in thrombus formation; this is the first such case to be reported. The complication was successfully treated by means of direct anastomosis following partial resection of the injured iliac artery. This is the first report of a case in which applying angiopressure for a vascular injury during laparoscopic pelvic lymphadenectomy led to an intra-arterial thrombus which was found and treated without sequelae.
\end{abstract}

\section{Introduction}

Minimally invasive laparoscopic surgery has become the mainstream surgical intervention worldwide. The field of gynecologic surgery is no exception, as minimally invasive gynecology has gained enormous momentum within the specialty. Although laparoscopy is considered a safe technique, it is not free of complications, with some complications resulting in fatalities. The most dangerous complication following insufflation is major vascular injury. Retrospective studies have reported the incidence of vascular injuries to range from $0.3 \%$ to $1.0 \%[1,2]$, whereas other studies have reported an incidence ranging from $0.04 \%$ to $0.5 \%$ [3].

Here, we report a case of thrombosis of the right iliac artery caused by an injury to the right external iliac artery, which occurred while the lymph node was being dissected during laparoscopy. The complication was successfully managed entirely by means of direct anastomosis following partial resection of the injured iliac artery. To our knowledge, this is the first report of a case in which applying angiopressure for a vascular injury during laparoscopic pelvic lymphadenectomy led to an intra-arterial thrombus which was found and treated without sequelae.

\section{Case Presentation}

A 53-year-old woman with a history of endometrioid carcinoma visited our hospital to be examined and treated for carcinoma. Following physical examination, magnetic resonance imaging (MRI), and a computed tomography (CT) scan (Figure 1), she was diagnosed with stage 1A endometrial cancer; thus, we decided to perform laparoscopic surgery which included total laparoscopic hysterectomy, bilateral salpingo-oophorectomy, and bilateral pelvic lymphadenectomy. Unfortunately, during surgery, we drew the penetrating branch during the right pelvic lymphadenectomy, causing a 


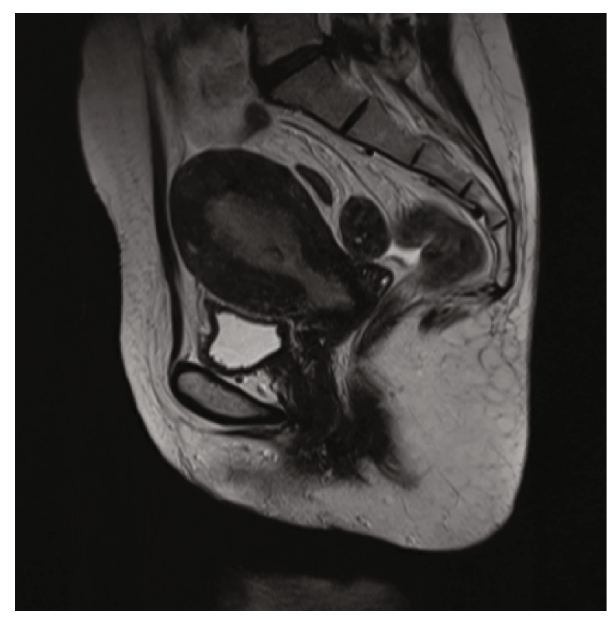

FIGURE 1: Magnetic resonance imaging of the pelvis. T2-weighted imaging revealing a thickened endometrium with a tumor.

$2 \mathrm{~mm}$ tear on the external iliac artery wall (Figure $2(\mathrm{a})$ ). We delivered continuous pressure to the injured site in the right iliac artery with small sterile absorbent gauze to stop the bleeding and prevent blood loss. With angiopressure continually being delivered, the left lymph node dissection was performed. After the dissection, the compression site was confirmed once again, and we observed rebleeding at the site where hemostasis had been previously achieved. After applying pressure to control the bleeding, TachoSil ${ }^{\circledR}$, made from fibrin and used as a hemostat, was applied to the bleeding site (Figure 2(b)). The duration of the entire surgery was 4 hours and 11 minutes.

Anticoagulation therapy such as low-molecular-weight heparin was not performed for this patient during 24 hours after surgery. In our hospital, anticoagulation therapy to prevent malignancy related is always started after 24 hours following the completion of surgery. This patient also was not applicable for preanticoagulation therapy, so she had not took pre- and postanticoagulation therapy.

On the day following the surgery, the patient developed right lower limb anterior leg pain, right lower limb coldness, and poor palpation in dorsal artery of the foot. Subsequently, enhanced CT was performed, and right external iliac artery thrombosis was confirmed. Concurrently, we performed surgery to remove a blood clot via the retroperitoneal approach by making a small incision and resolved the thrombosis by suturing the artery following resection of the injured external artery (Figures 3-5).

\section{Discussion}

In this report, we describe a unique case of endometrial cancer in a patient who suffered a vascular injury while undergoing laparoscopic lymph node dissection. First, we applied continuous pressure at the site of injury using Trox gauze ${ }^{\circledR}$ to control bleeding and then used TachoSil ${ }^{\circledR}$ to stop the bleeding. On postoperative day 1 , the patient reported right lower limb anterior leg pain together with lower limb coldness; we confirmed thrombus formation based on the vascular injury and artery pressure.

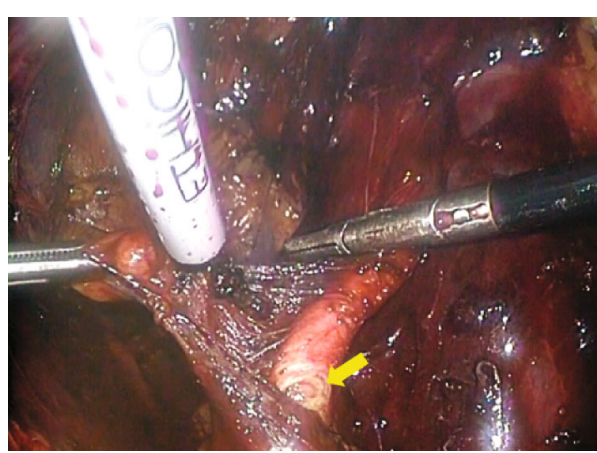

(a)

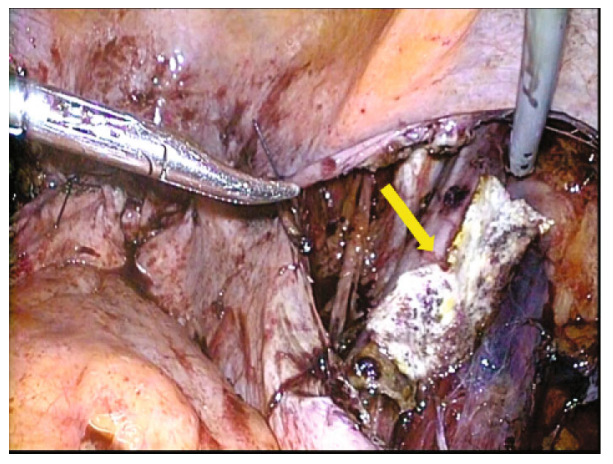

(b)

FIGURE 2: (a) Laparoscopic view of the iliac artery injury (arrow). (b) Laparoscopic view of the repaired iliac artery after using TachoSil (arrow).

Laparoscopic surgery is generally considered a safe surgical procedure. Additionally, advanced laparoscopic techniques have been recently used to treat endometrial, cervical, and ovarian malignant disease $[1,4,5]$. Laparoscopic lymphadenectomy is an oncologically safe and standardized technique [2]: the rate of vascular injury in laparoscopic lymphadenectomy is comparable to that in conventional laparotomy, and the injury is usually reparable using a laparoscopic technique. Previous studies report that major vascular injuries occur in $0.07-4.46 \%$ of patients undergoing laparoscopic surgery for endometrial cancer [6-9]. Based on a multicenter study of the largest number of laparoscopic lymphadenectomy cases, Chapron et al. reported that major vascular injury occurred in $0.02 \%$ of the cases [10].

The first step to control massive bleeding is to apply direct pressure over the bleeding site with the aid of a sponge stick, which will provide additional time for repairing lacerated vessels.

Previously, several authors have reported cases of compartment syndrome resulting from a thrombus during gynecologic surgery [11]. However, few reports describe thrombosis occurring in artery vessels as a result of applying angiopressure. To our knowledge, this is the first report of a case in which applying angiopressure for a vascular injury during laparoscopic pelvic lymphadenectomy led to an intra-arterial thrombus which was found and treated without sequelae.

Drawing penetrating branch is a vessel injury that may occur during lymphadenectomy. The injury usually results in a small tear and is controlled by applying angiopressure. 


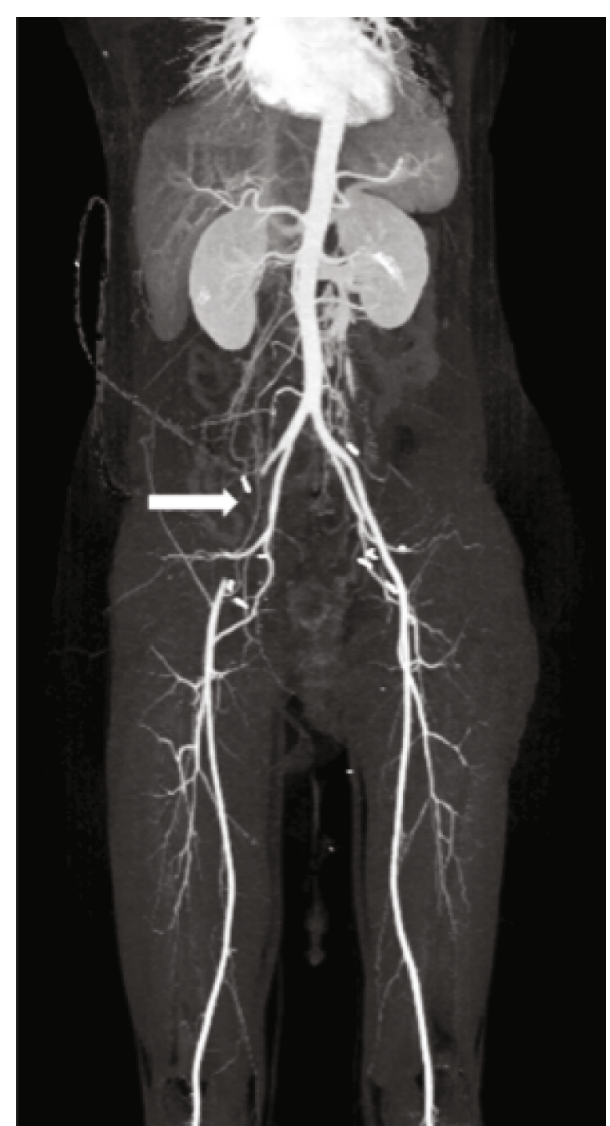

FIGURE 3: Axial contrast-enhanced computed tomography indicating occlusion of nearly the entire right common iliac artery (arrow).

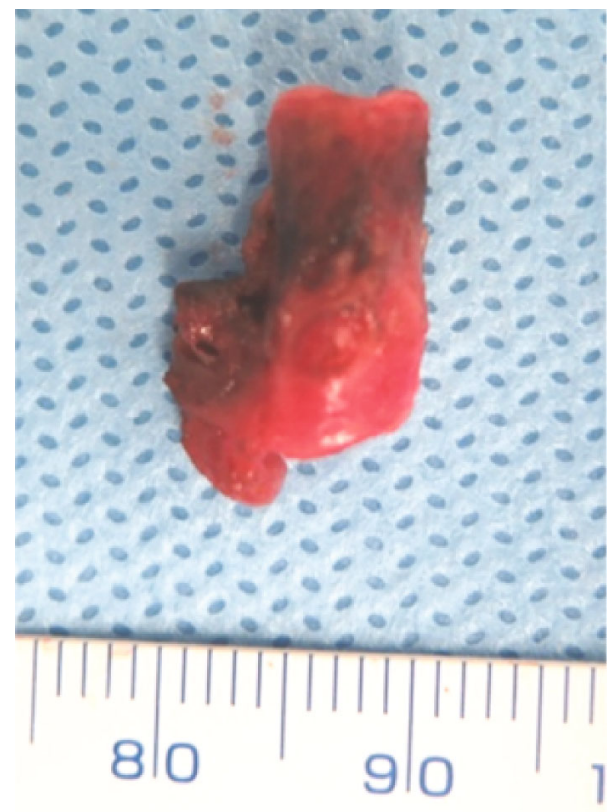

FIGURE 4: Operative findings. Thrombosis is noted inside the artery.

In this case, the injury was slightly larger than commonly reported, while angiopressure was applied for a longer period than necessary, which is what might have caused thrombosis

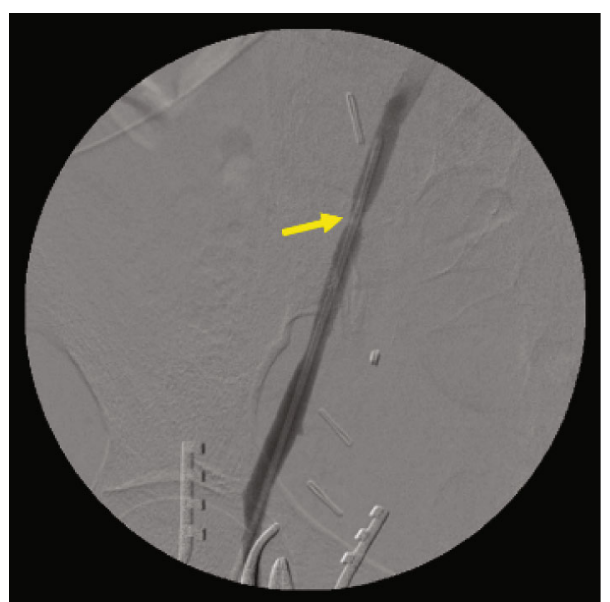

FIgURE 5: Fluoroscopy X-ray image shows contrast dye that passed through the artery following suture (arrow).

in the artery. The case presented herein confirms that applying pressure on an injured vessel may lead to thrombosis, which thus complicates the patient's condition. Here, we were able to overcome this problem by suturing the arteries following a $2 \mathrm{~cm}$ resection of the injured artery. It would be wise for a physician to treat iliac artery injury by performing direct anastomosis subsequent to surgical repair of the damaged vessel.

In this case report, besides presenting the unusual clinical symptoms of a patient following surgery, we want to emphasize that lower limb pain and coldness could be an early sign of thrombosis. Early recognition of these symptoms is essential for providing prompt and appropriate treatment and improving the patient's overall prognosis.

In summary, this report describes an unusual vascular injury that occurred during laparoscopy and highlights the importance of suturing vessels immediately. Moreover, in the case of unusual clinical symptoms such as pain and coldness following surgery, physicians should check for thrombosis and provide adequate treatment.

\section{Data Availability}

Not applicable.

\section{Conflicts of Interest}

The authors declare no conflicts of interest. Submitting authors are responsible for coauthors declaring their interests.

\section{Authors' Contributions}

Masako Ishikawa and Kentaro Nakayama drafted the manuscript. Hitomi Yamashita, Tomoka Ishibashi, Seiya Sato, Hiroki Sasamori, Kiyoka Sawada, Sonomi Kurose, and Shoichi Suehiro helped correcting the patient's date. Noriyoshi Ishikawa carried out the pathological diagnosis. Sultana Razia corrected the manuscript. Satoru Kyo conceived the study, participated in its design and coordination, and helped in drafting the manuscript. All authors have read and approved the final manuscript. 


\section{Acknowledgments}

The authors wish to thank the Division of Cardiovascular and Thoracic Surgery, Department of Surgery, and the Department of Pathology for supporting this work.

\section{References}

[1] A. G. Nordestgaard, K. C. Bodily, R. W Osborne Jr., and J. D. Buttorff, "Major vascular injuries during laparoscopic procedures," The American Journal of Surgery, vol. 169, no. 5, pp. 543-545, 1995.

[2] S. Sandadi, J. A. Johannigman, V. L. Wong, J. Blebea, M. D. Altose, and W. W. Hurd, "Recognition and management of major vessel injury during laparoscopy," Journal of Minimally Invasive Gynecology, vol. 17, no. 6, pp. 692-702, 2010.

[3] D. S. Seidman, F. Nasserbakht, F. Nezhat, C. Nezhat, and C. Nezhat, "Delayed recognition of iliac artery injury during laparoscopic surgery," Surgical Endoscopy, vol. 10, no. 11, pp. 1099-1101, 1996.

[4] P. B. Panici, F. Plotti, M. A. Zullo et al., "Pelvic lymphadenectomy for cervical carcinoma: laparotomy extraperitoneal, transperitoneal or laparoscopic approach? A randomized study," Gynecologic Oncology, vol. 103, no. 3, pp. 859-864, 2006.

[5] C. Köhler, P. Klemm, A. Schau et al., "Introduction of transperitoneal lymphadenectomy in a gynecologic oncology center: analysis of 650 laparoscopic pelvic and/or paraaortic transperitoneal lymphadenectomies," Gynecologic Oncology, vol. 95, no. 1, pp. 52-61, 2004.

[6] C. C. Gunderson, J. Java, K. N. Moore, and J. L. Walker, "The impact of obesity on surgical staging, complications, and survival with uterine cancer: a Gynecologic Oncology Group LAP2 ancillary data study," Gynecologic Oncology, vol. 133, no. 1, pp. 23-27, 2014.

[7] J. D. Wright, D. L. Hershman, W. M. Burke et al., "Influence of surgical volume on outcome for laparoscopic hysterectomy for endometrial cancer," Annals of Surgical Oncology, vol. 19, no. 3, pp. 948-958, 2012.

[8] S. Kondalsamy-Chennakesavan, M. Janda, V. Gebski et al., "Risk factors to predict the incidence of surgical adverse events following open or laparoscopic surgery for apparent early stage endometrial cancer: results from a randomised controlled trial," European Journal of Cancer, vol. 48, no. 14, pp. 21552162, 2012.

[9] F. Zullo, S. Palomba, A. Falbo et al., "Laparoscopic surgery vs laparotomy for early stage endometrial cancer: long-term data of a randomized controlled trial," American Journal of Obstetrics and Gynecology, vol. 200, no. 3, pp. 296.e1-296.e9, 2009.

[10] C. Chapron, D. Querleu, M. A. Bruhat et al., "Surgical complications of diagnostic and operative gynaecological laparoscopy: a series of 29,966 cases," Human Reproduction, vol. 13, no. 4, pp. 867-872, 1998.

[11] E. C. A. Bauer, N. Koch, C. J. Erichsen et al., "Survey of compartment syndrome of the lower extremity after gynecological operations," Langenbeck's Archives of Surgery, vol. 399, no. 3, pp. 343-348, 2014. 